\title{
AN AUDIT OF REFERRALS WITH HEADACHES TO PAEDIATRIC NEUROLOGY AT SHEFFIELD CHILDREN'S HOSPITAL
}

\author{
N. de Vere, S. Martin, S. Mordekar \\ Paediatric Neurology, Sheffield Children's Hospital, Sheffield, UK
}

Background and aims: Headaches are common in children and in most cases it is simply advice and reassurance that is required. Referrals to paediatric neurology in Sheffield come from general practice, and local and regional general paediatricians. We audited whether headaches were being managed in accordance with recognised guidance.

Methods: We obtained a list of 73 patients referred with headache to the three paediatric neurologists by searching clinic letters filed on the network. We then reviewed the case notes of 71 of these (two were excluded as headache was not the primary problem.) A proforma was designed to record patient demographics, history, examination findings, investigations, management and follow-up.

Results: The mean age at first out-patient visit was 11.5years (range 4-17 years.) The majority of cases came from general practice (41\%.) $31 \%$ of children were not using simple analgesia at the time of referral. History taking was satisfactory. Documentation of examination findings was present in $100 \%$ of cases but blood pressure was only recorded in $69 \%$ of cases. MRI/CT was done in 32 cases $(45 \%)$. In the majority of cases no medications were prescribed following appointment but documentation of advice given was very poor; $33 \%$ of cases.

Conclusions: The history taking and diagnoses given were in line with current best practice. Blood pressure measurement is poor and although this is a common problem in paediatrics it is particularly surprising in a paediatric neurology service. Advice was not well documented and as this may be leaving families dissatisfied. 